Meta

Journal des traducteurs

Translators' Journal

\title{
Pistes pour une nouvelle approche de la traduction automatique des noms propres
}

\section{Jean Louis Vaxelaire}

Volume 51, numéro 4, décembre 2006

La traduction des noms propres (1) et Langue, traduction et mondialisation : interactions d'hier, interactions d'aujourd'hui Language, Translation and Globalization: Interactions from Yesterday, Interactions from Today (2)

URI : https://id.erudit.org/iderudit/014337ar

DOI : https://doi.org/10.7202/014337ar

Aller au sommaire du numéro

Éditeur(s)

Les Presses de l'Université de Montréal

ISSN

0026-0452 (imprimé)

1492-1421 (numérique)

Découvrir la revue

Citer cet article

Vaxelaire, J. L. (2006). Pistes pour une nouvelle approche de la traduction automatique des noms propres. Meta, 51(4), 719-738.

https://doi.org/10.7202/014337ar
Résumé de l'article

Qu'est-ce que les noms propres ? Sont-ils traduisibles ? Une réponse claire à ces deux questions semble préalable à toute tentative de traitement automatique des noms propres. Certains auteurs prétendent qu'ils sont intraduisibles, alors que la pratique prouve le contraire.

Divers critères tels que le genre textuel, le contexte historique, la langue source ou la nature ontologique du porteur jouent un rôle important dans la décision de modifier ou de conserver dans sa forme originale un nom propre. 


\title{
Pistes pour une nouvelle approche de la traduction automatique des noms propres
}

\author{
JEAN LOUIS VAXELAIRE \\ Université Paris VII, Paris, France \\ jl.vaxelaire@wanadoo.fr
}

\begin{abstract}
RÉSUMÉ
Qu'est-ce que les noms propres? Sont-ils traduisibles? Une réponse claire à ces deux questions semble préalable à toute tentative de traitement automatique des noms propres. Certains auteurs prétendent qu'ils sont intraduisibles, alors que la pratique prouve le contraire.

Divers critères tels que le genre textuel, le contexte historique, la langue source ou la nature ontologique du porteur jouent un rôle important dans la décision de modifier ou de conserver dans sa forme originale un nom propre.
\end{abstract}

\begin{abstract}
What are proper names? Are proper names translatable? A clear answer to those two questions should be prior to any attempt of machine translation of the proper names. Some authors claim that the proper name is untranslatable, while the practice shows the opposite.

Different criteria such as the textual genre, the historic context, the source language or the ontological nature of the bearer play a significant role in the decision to modify or to preserve the proper name in its original shape.
\end{abstract}

MOTS-CLÉS/KEYWORDS

contexte, culture, nom propre, traduction automatique, typologie

\section{INTRODUCTION}

À lire les supports promotionnels des systèmes de traduction automatique disponibles pour le grand public, le traitement des noms propres a longtemps été considéré comme un problème mineur, avant de devenir un réel enjeu. Naïvement, le critère de la majuscule initiale semblait idéal pour localiser le nom propre, du moins dans le couple français-anglais. Il était en revanche moins efficace dans d'autres couples de langues, en particulier dans des langues dont les alphabets ne comportent pas de majuscules (c'est-à-dire tous les systèmes d'écriture qui ne sont pas originaires d'Europe). Une fois localisé, il fallait alors l'« exclure» ou le « réserver» pour éviter que George Bush ne devienne George Buisson. Les défauts de cette approche ont heureusement été perçus et les chercheurs travaillent aujourd'hui sur d'autres options.

Pourtant, avant de penser à demander à des programmes de gérer la traduction des noms propres, il faut être conscient que le problème de la traduction «classique» des noms propres n'est pas réglé, le débat est encore encombré par de trop nombreux lieux communs. Le premier est qu' « un nom propre ne se traduit pas; il ne peut, à la limite, que s'adapter» (Rey 1979: 28). Pourtant, comme le montre Dauzat (1928: 156), les noms furent longtemps traduits (lorsqu'ils avaient un sens apparent) ou fortement francisés. 
Réduire le passage des noms propres d'une langue à une autre à une simple adaptation indique une vision trop étroite du phénomène. S’il est vrai que Vienne est une naturalisation du nom allemand Wien, ce n'est pas le cas de United Kingdom qui devient le Royaume-Uni en français. Nous considérerons donc que les deux phénomènes coexistent, bien que l'on puisse envisager à la manière de Josette Rey-Debove (1969: 128) que nous avons dans tous les cas des adaptations (grapho-phonique, phonique, et même aussi sémantique comme dans le cas du calque). Cette option est cependant rejetée par Algeo (1973: 60) pour qui, dire que Vienna et Germany sont les versions anglaises de Wien et Deutschland plutôt que des traductions revient à ergoter sur des questions de terminologie ${ }^{1}$.

Nous parlerons donc pour les exemples de naturalisation d'adaptation $\mathrm{du}$ nom propre et dans ceux du second, de traduction $d u$ nom propre (ce terme regroupe les calques du type Royaume-Uni mais aussi les cas mixtes comme Pierre le Grand). Enfin, les termes d'adaptation et de traduction ne suffisent plus dans le cas du Golfe de Gascogne qui s'appelle Bay of Biscay en anglais et Mar Cantabrico en espagnol. Chaque langue a en fait choisi une dénomination qui lui est propre. Nous parlerons dans ce cas de dénomination multiple 2 .

La définition du nom propre demeure un autre problème crucial. De nombreux auteurs parlent de noms propres sans en donner une définition précise, comme si elle était évidente. Malheureusement, lorsque l'on parcourt la littérature sur le sujet, on se rend compte que cette définition n'est pas identique chez tous les auteurs, aussi bien en intension qu'en extension. De plus, on trouve trop souvent une vision très simpliste du nom propre. Ainsi, la philosophe analytique américaine, en l'occurrence Salmon (1989: 211), parle des «proper names or other simple (non-compound) singular terms» comme si les premiers étaient obligatoirement simples! On constate finalement que la plupart des philosophes et des linguistes considèrent les noms propres comme des formes simples comprenant un ou deux termes au maximum (c'est pourquoi Socrate demeure un exemple particulièrement prisé).

Nous renvoyons à un autre travail (Vaxelaire 2001) pour une description plus approfondie, mais nous considérerons ici qu'un nom propre est un signe référant à un individu de manière récurrente dans un texte ou un corpus, sa forme pouvant aller d'une simple lettre à une phrase complète.

Nous nous intéresserons tout d'abord aux conditions qui amènent à modifier ou à conserver un nom propre dans la langue cible à l'écrit. Contrairement à Agafonov et al. (dans ce numéro), nous ne considérons pas que Washington ou Myanmar soient des emprunts, mais des formes originales conservées à l'écrit. Lorsque le gouvernement du Myanmar impose qu'on ne modifie pas son nom, il ne demande pas qu'on lui «emprunte» mais refuse une quelconque naturalisation.

Nous verrons ensuite si les principaux types de noms propres sont le plus souvent modifiés ou conservés.

\section{MODIFICATION OU CONSERVATION DANS LA LANGUE CIBLE?}

\section{II.1. Le rôle du contexte}

Hartwig Kalverkämper (1995: 1021-1024) a clairement montré que le choix de modifier ou de conserver un nom propre dans la langue cible était souvent dicté par le type 
de support. Ainsi, on accompagne parfois dans les textes didactiques les noms propres non traduits de notices étymologiques: dans un article de dictionnaire sur Lénine, on voit entre parenthèses les traductions de Iskra et Pravda. Kalverkämper conclut qu'on traduira plus souvent les noms dans ces textes didactiques, dans la littérature enfantine (ex.: La Belle au Bois dormant / The Sleeping Beauty / Dornröschen et Le Chat botté / Puss in Boots / Der gestiefelte Kater) et dans les contextes interculturels que dans un reportage d'information bien qu'il existe quelques contre-exemples, comme dans l'édition du 7 septembre 2001 de Courrier International: "À Belfast, la rentrée des classes s'accompagne, pour les fillettes inscrites à l'école Sainte-Croix ${ }^{3}$, d'une redoutable épreuve quotidienne.»

Dans les travaux universitaires, certains noms propres sont uniquement employés pour leur valeur culturelle et les traducteurs peuvent donc les remplacer par des références locales qui ne déconcentreront pas le lecteur: les Socrates, Guy Fawkes et Harold Wilson de A Short History of Linguistics de Robins deviennent ainsi Socrate, Ravaillac et Pompidou dans la version française (Brève histoire de la linguistique - De Platon à Chomsky, Paris, Seuil, 1976: 82). Le procédé est identique lorsque l'auteur choisit certains noms pour leur valeur ethnique: les Karl Müller, Max Östreicher, Paul Mendelssohn et le tailleur Schulze des Prinzipien der Sprachgeschichte de Hermann Paul ont été remplacés par des noms typiquement anglais dans la traduction anglaise (Principles of the History of Language, College Park, McGrath, 1970: 320), respectivement John Smith, Peter Robinson et Henry English et le tailleur Thomson. Que le nom soit celui d'une célébrité ou inventé ad hoc, il n'est pas en lui-même primordial, il sert uniquement à illustrer un raisonnement.

\section{II.2. Une question historique}

Conserver un nom propre dans sa forme originale aurait été une hérésie dans toute l'Antiquité. L'un des premiers traducteurs qui ont choisi de ne pas modifier les noms de lieux et de personnes semble être Sir John Trevisa, pour sa traduction anglaise du Polychronicon de Ralph Higden en 1387 (Hermans 1988: 11). D’après Maillot (1979: 211), la tendance à naturaliser les noms étrangers a en effet commencé à disparaître avec l'abandon du latin au profit des langues vernaculaires. Demonet (1992) en voit également la trace dans le courant «idiomatiste» qui se développe dès le début du $\mathrm{XVI}^{\mathrm{e}}$ siècle, par exemple le Veteris Testamenti ad veritatem Hebraicam recognitio de Steuco (publié à Venise en 1529) qui préfère laisser les noms propres tels quels. Ainsi que l'a remarqué Schultze (1991: 107), à partir de la seconde moitié du XIX ${ }^{e}$ siècle et durant tout le $\mathrm{xx}^{\mathrm{e}}$ siècle, la tendance de ne pas modifier les noms propres prit de plus en plus d'importance.

Les adaptations ou les traductions posent aujourd'hui les mêmes problèmes que les néologismes et les xénismes: certaines demeurent et d'autres disparaissent. Par exemple, la version française de Brutus (Brute) n'a pas été conservée, contrairement à la majorité des noms latins ou grecs (Jules César, Socrate, Virgile, Achille ou Plato, Livy, Aquinas, Epictetus en anglais). Bien qu'elle ait également disparu, il existait au $\mathrm{XVII}^{\mathrm{e}}$ siècle une forme francisée de Buckingham qui s'écrivait Bouquinquan (Dauzat 1977; Carton, 1968).

Les surnoms ont toujours été traduits ${ }^{4}$ (Røde Erik / Eric le Rouge; John Lackland / Jean Sans-terre; Pietro il Grande / Peter the Great / Pierre le Grand), mais on a également 
pu traduire des patronymes: le peintre flamand Rogier Van der Weyden devenait Roger de la Pasture pour les francophones; les humanistes traduisaient leur nom en grec ou en latin (Michel de Notre-Dame est aujourd'hui plus connu sous le nom de Nostradamus, Jacques Dubois est devenu Jacobus Sylvius, Schwarzerd est devenu Melanchton et Reuchlin a créé une forme grecque Capnion en jouant sur son nom: Rauch $=$ «umée $»)$.

M. Fourment-Berni Canani a très justement remarqué que «la re-nomination [ce que nous appelons ici traduction ou adaptation] est un fait culturel qui a évolué avec l'histoire» (1994: 560). On parlera ainsi de Gustave de Suède mais pas de Jean Kennedy et London, la ville canadienne, ne devient pas Londres en français ou Londra en italien ${ }^{5}$. On pourrait ajouter que Venice en Californie, Athens en Géorgie, Cordoba en Argentine ou Toledo en Ohio ne se transforment pas non plus en Venise, Athènes ${ }^{6}$, Cordoue ou Tolède. Ces villes créées par les colons demeurent généralement dans leur langue d'origine, on a certes autrefois traduit Nieuwe Amsterdam par Nouvelle-Amsterdam mais ce n'est plus le cas de New York. Pour rester dans les exemples avec New, on peut voir que les usages sont variables: certains noms d'États américains ne sont pas modifiés (New Jersey ou New Hampshire, contrairement au Nouveau-Mexique) alors que les noms de pays ou de régions le sont (New Zealand / Nouvelle-Zélande, New England / Nouvelle-Angleterre). Ces différences existent dans d'autres langues comme le tchèque qui parle de New York et de Nový Zéland (Elman 1986: 27). Spitzer (1937: 326) avait fait remarquer qu'en 1937, les Hongrois écoutaient les musiques de Mozart Farkas et Beethoven Lajos. Les noms ont en fait longtemps été adaptés à la manière hongroise (le patronyme précédant le prénom), Jules Verne et Jean Calvin sont donc devenus selon l'ancienne méthode Verne Gyula et Kálvin János mais Jean Cocteau, plus contemporain, est demeuré Jean Cocteau ${ }^{7}$ (Kálmán 1978: 12). Jusque dans les années 1960, on trouvait en Italie les livres de Guglielmo Shakespeare, Federico Schiller et Gian Giacomo Rousseau (Manini 1996: 171). Dans le Novissimo dizionario della lingua italiana (1940, c'est-à-dire durant la période mussolinienne), Fernando Palazzi écrit même (p. 1315) qu'il est honteux de ne pas employer les versions italiennes des noms propres étrangers ${ }^{8}$ (Ibid.: 172). Lors de sa première publication française en 1796, Hermès était l'œuvre de Jacques Harris. Dans sa réédition de 1972, l'auteur s'appelait James Harris.

Il n'est même pas nécessaire de prendre des exemples aussi anciens pour montrer ces différences de traitement selon les époques: les titres de films américains ou anglais sont de moins en moins souvent traduits alors qu'ils l'étaient systématiquement jusqu'aux années 1960/1970. Dans le domaine littéraire, le traducteur de La prochaine fois, le feu / The Fire Next Time de Baldwin a modifié Nation of Islam (Nation d'Islam) et Black Muslims (Musulmans noirs). Cette traduction date de 1963 et il est fort probable qu'aujourd'hui, on ne toucherait plus à ces noms car ils ne sont plus traduits dans la presse.

E. Pichon aurait souhaité que l'on continue à traduire tous les noms étrangers car le système de non-traduction introduit, selon lui, « de la confusion dans la géographie» (1937: 114). Ainsi, en 1937, personne, à part les Slovaques, n'appelle la ville de Košice Košice: il s'agissait de Kassa pour les Hongrois, de Kaschau pour les Allemands et de Cassovie pour les Français. Les usages ont changé, on essaie aujourd'hui de garder au maximum les formes originales. Si l'on ne tente pas de remplacer en français Londres par London, il n'en demeure pas moins que peu de 
personnes utilisent encore de nos jours Cassovie pour désigner Košice (le PR2 ignore cette version alors qu'il donne les versions allemande et hongroise citées plus haut). Tout comme Cassovie, les francophones semblent actuellement abandonner, quelquefois par ignorance, certaines formes comme Brousse pour la ville de Bursa en Turquie, Presbourg pour la capitale slovaque Bratislava ou L'Écluse pour Sluis aux Pays-Bas?. Les formes naturalisées les plus populaires sont donc conservées ${ }^{10}$ et les moins courantes semblent être abandonnées au profit des formes originales.

Toutefois, comme le fait remarquer T. Grass (dans ce numéro), les anciennes formes naturalisées doivent être employées dans des contextes historiques. Ainsi, bien que l'on utilise aujourd'hui la forme polonaise Wroclaw en allemand, on continue à employer Breslau lorsque l'on parle de cette ville dans l'entre-deux-guerres. De même, il serait incongru dans un texte consacré à Kant de remplacer Königsberg par sa forme actuelle Kaliningrad ${ }^{11}$.

\section{II.3. Des différences marquées selon la langue source}

Pour se rendre compte des différences de traitement selon la langue source, nous avons étudié comment les guides touristiques (en l'occurrence les Guides Verts de Michelin) traduisent les noms des sites qu'ils décrivent. Nous avons pensé qu'il n'était pas nécessaire de prendre des exemples de langues très exotiques ou possédant d'autres systèmes d'écriture, la comparaison avec des langues européennes pouvait être suffisamment éclairante. Nous avons donc étudié les noms flamands du guide de la Belgique, le guide du Danemark, celui de l'Espagne et enfin celui de la Grande-Bretagne. Les noms des auteurs ne sont pas indiqués, il y en a probablement plusieurs par guide - ce qui expliquerait les différences de traitement au sein d'un même ouvrage.

En ce qui concerne les musées par exemple, les différences sont saisissantes. Les noms de musées danois sont presque systématiquement traduits:

Nationalmuseet (Copenhague) $=$ Musée national;

Fyns Kunstmuseum (Odense) = musée fionien des Beaux-Arts;

Danmarks Cykelmuseum (Ålestrup) = musée danois de la Bicyclette;

Vikingsmuseet $($ A rhus $)=$ Musée viking;

Ålborgs Historiske Museum (Alborg) = musée d'Histoire;

Kunstmuseum (Silkeborg, Århus) = musée des Beaux-Arts.

Les deux seuls musées dont les noms ne sont pas traduits sont assez transparents puisqu'il s'agit du Post \& Tele Museum de Copenhague et du Skagens Museum de Skagen.

La situation est similaire dans le guide belge, où tous les noms de musées sont traduits sauf quelques-uns comprenant un anthroponyme (le Museum Leonardo da Vinci de Tongerlo ou le Memlingmuseum de Bruges par exemple):

Museum voor Volkskunde (Bruges, Gand) = Musée du Folklore;

Etnografisch museum (Anvers) = Musée d'Ethnographie;

Museum voor Schone Kunsten (Gand) = Musée des Beaux-Arts;

Zuidwest Brabants Museum (Hal) = Musée du Sud-Ouest du Brabant;

Nationaal Jenevermuseum (Hasselt) = Musée national du Genièvre;

Herinneringsmuseum (Ypres) $=$ Musée du Souvenir;

Nationaal Visserijmuseum (Coxyde) = Musée national de la Pêche;

Gallo-Romeins Museum (Tongres) = Musée gallo-romain . 
Par contre, lorsque l'on passe à des langues latines comme l'espagnol ou le catalan, la situation est loin d'être identique. Les traductions sont bien plus rares, elles sont même l'exception:

Museu Téxtil i d'Indumentària (Barcelone) = musée du Textile et du Vêtement;

Museu Maritim (Barcelone) = musée de la Marine;

Museo de Telas Medievales (Burgos) = musée des Tissus médiévaux.

Les autres musées sont uniquement donnés dans leur version originale ${ }^{12}$, qu'elle soit transparente (les différents Museo de Bellas Artes de Bilbao, La Corogne, Vitoria, Malaga, Santander ou Séville, Museu de Zoologia de Barcelone ou encore Museo Diocesano de Arte Sacro de Bilbao) ou qu'elle ne le soit pas obligatoirement pour un Français (Museo del Ferrocarril à Madrid, Museu de Joguets à Figueras ou Museo Vasco à Bilbao). On peut en effet penser qu'un francophone risque plutôt de voir dans ce Museo Vasco un hommage à Vasco de Gama qu'au pays Basque.

Les proportions entre traduction et non-traduction des noms de musées sont grossièrement équivalentes entre le guide sur l'Espagne et celui sur la GrandeBretagne:

Colour Museum (Bradford) = musée de la Couleur;

Musée de la Vie rurale (Glastonbury) = Somerset Rural Life Museum;

National Motor Museum (Beaulieu) = musée national de l'Automobile;

D-Day Museum (Portsmouth) = musée du Débarquement;

Ripon Workhouse Museum of Yorshire Poor Law (Ripon) = musée de la maison de correction de Ripon;

Museum of British Road Transport (Coventry) = musée des Transports routiers anglais ${ }^{13}$.

Comme le touriste francophone parti en Espagne, celui qui se rend en GrandeBretagne se retrouve face à des noms de musées sans traduction qui peuvent parfois être transparents (Gallery of Modern Art à Glasgow, Canal Museum près de Northampton, British in India Museum à Colne ou British Photographic Museum à Totnes) mais qui, dans de nombreux cas, ne le sont pas sans une certaine connaissance de l'anglais. Ainsi, les non-traductions du Lock Museum de Willenhall, du Scotland's Lighthouse Museum de Fraserburgh, du Guildhall Museum de Boston ou encore du Cregneash Folk Museum (situé sur l'île de Man) sont surprenantes. Les musées de Londres (British Museum, National Gallery, Natural History Museum, National Maritime Museum, Science Museum) font partie de ces noms sans équivalents en français.

Ces traitements différents entre l'anglais et l'espagnol d'un côté, le flamand et le danois de l'autre, se retrouvent avec les autres types de noms. Les noms d'églises (Vor Frue Kirke de Copenhague, Kalundborg, Nyborg, Svendborg et Århus = église NotreDame) ou de châteaux danois (Egeskov Slot = Château d'Egeskov; Frederiksborg Slot = Château de Frederiksborg; Rosenborg Slot = Château de Rosenborg, etc.) sont systématiquement associés à leurs équivalents français alors que les châteaux anglais sont traduits dans certains cas mais peuvent aussi ne pas l'être. Ainsi, sur une même page, on peut voir Floors Castle (sans traduction) et Château de Norham ou encore Lindisfarne Castle (également sans traduction) et Château de Bamburgh. En effet, dans les guides anglais et espagnols, si certains noms sont traduits (Holy Trinity Church = église de la Sainte-Trinité; Callanish Standing Stones = mégalithes de Callanish; Barri Gòtic $=$ Quartier gothique; Palacios nazaries = palais nasrides $)$, de nombreux autres ne 
le sont pas (Flag Fen Bronze Age Excavation, The Historic Dockyard, Fountains Abbey, Palau de la Generalitat, Claustro de los Caballeros). Dans le même temps, les noms danois ou flamands sont pratiquement toujours traduits (Rådhuset $=$ hôtel de ville et Marmorkirken = église de marbre pour le danois; Stadhuis = Hôtel de ville et Begijnhof = Béguinage pour le flamand). L'opposition est encore plus forte entre la Plaza Mayor (de Trujillo, Burgos, Madrid ou Salamanque) qui n'est jamais traduite, et la Grote Markt (de Courtrai, Alost, Anvers ou Malines) qui est systématiquement associée à Grand-Place.

Il nous semble que les auteurs de ces guides estiment que les Français ont des notions d'anglais et d'espagnol (par exemple lorsqu'il est écrit à propos de la Calle Mayor de Madrid que «son nom rappelle l'importance de cette rue» alors qu'il n'est donné aucune version française, et que ces bases sont inexistantes en flamand ou en danois. Un rapide coup d'œil sur le guide consacré à l'Europe confirme ces différences: les toponymes grecs, hongrois, polonais ou roumains sont généralement traduits, au contraire de leurs homologues irlandais ou italiens.

Ces différences selon la langue source se répercutent dans tous les domaines. J. Humbley (2000) avait noté qu' «on traduit systématiquement en français les noms des institutions scandinaves ou slaves, mais on laisse souvent dans la langue d'origine les organismes de langue anglaise ou, moins souvent, latine». Cette pratique se retrouve également dans les sous-titres du film In the Mood for Love (Hong-Kong, 2000) où un titre de chanson chinoise est traduit en français alors que les titres des chansons angloaméricaines ne le sont jamais. On s'aperçoit que les noms issus de langues «exotiques» (l'exotisme débutant avec les langues anglo-saxonnes autres que l'anglais) seront presque inévitablement modifiés. On sait que le nom du syndicat polonais Solidarnosc a souvent été traduit dans les années 1980 par Solidarité avant d'acquérir une légitimité dans sa forme originale. On peut douter qu'un syndicat anglais appelé Solidarity aurait vu son nom traduit.

L'usage est identique dans la littérature scientifique. Pour ne prendre que deux exemples, M. Yaguello (Les Fous du langage, Seuil, Paris, 1984) cite les titres de livres russes en traduction française alors qu'elle ne modifie pas ceux des ouvrages en anglais et Beci (2001) traduit en français les références albanaises de sa bibliographie.

Enfin, les journalistes pratiquent le plus souvent une discrimination positive envers l'anglais. Ainsi, on reste sceptique devant le German Federal Institute for Risk Assessment (qui est d'après le Canard enchaîné du 9 juillet 2003 «l'équivalent allemand de notre Agence française de sécurité sanitaire des aliments»). En effet, il nous semble douteux qu'une institution allemande ait un nom en anglais.

1. Beckenbauer [...] esquisse un léger sourire lorsqu'il entend le kop du Bayern reprendre une chanson intitulée On ne peut pas séparer les bons amis, que le Kaiser avait chantée lorsqu'il était encore joueur. À 22 h 25, M. Kircher siffle la fin de la rencontre. Au même moment, les lumières du stade Olympique s'éteignent pour laisser place durant près de dix minutes à un magnifique feu d'artifice, qui clôt un grand spectacle, tant sur le terrain que dans les tribunes, accompagné de la chanson FC Bayern forever Number One. (FF, $\mathrm{n}^{\circ} 2991,5$ août 2003)

2. «Promus au rang de lieutenant, ils sont incorporés à l'état-major de l'armée ou dans certaines unités de combat, notamment le long de la zone démilitarisée», explique le professeur Alexandre Mansourov, de l'Asia-Pacific Center for Security Studies, un organisme de recherche sur la sécurité basé à Hawaï. «En 1990, le nombre de personnes formées fut jugé suffisant pour créer une section spéciale dédiée à la guerre électronique 
auprès de l'état-major nord-coréen, l'Electronic Warfare Bureau.» (Le Monde, 23 juin 2003)

Dans ces deux exemples, on se trouve face à deux noms d'origine étrangère. Dans (1), la chanson au titre allemand se voit traduite, alors que la seconde, au titre anglais, ne l'est pas. Dans l'exemple suivant (2), s'il n'y a rien de choquant à ce que le nom de l'institut américain (Asia-Pacific Center for Security Studies) ne soit pas traduit, puisque cela suit l'usage actuel, il est par contre plus étonnant de voir l'état-major nordcoréen donner un nom anglais à l'une de ses sections (Electronic Warfare Bureau).

La difficulté de prononciation et d'écriture du nom d'origine est quelquefois prise en compte. Ainsi, les noms de clubs de football ne sont pas modifiés (Grasshopper Zürich, Real Sociedad, etc.), même si celui de la ville l'est (la F.C. Bayern München et le F.C. Barcelona deviennent le Bayern de Munich et le le F.C. Barcelone). Le seul nom de club que nous ayons vu traduit est celui du Crvena Zvezda Beograd qui est traduit par Étoile Rouge de Belgrade ou Red Star Belgrad en anglais). Puisque les autres clubs de la capitale de la Serbie-Monténégro (Partizan, Obilic, OFK, etc.) ne sont pas traduits, on peut donc penser que le fait que ce nom soit particulièrement compliqué pour les non-serbophones ait joué dans sa traduction.

Il serait faux de laisser croire que les noms propres anglais ne sont jamais traduits. Ils peuvent également l'être, principalement dans la littérature enfantine ou dans certains contextes particuliers, comme celui du Québec dont nous parlerons dans la prochaine partie.

La littérature pour adultes peut également parfois traduire quelques noms propres. On lit ainsi dans Etats sauvages de Stephen Wright qu'un personnage possède des magazines intitulés Moto-Passion, Fortune, Jouissances et qu'une femme ressemble à une actrice blonde de Braises ardentes ou Haine et passion ou Amour, gloire et beauté. Ces traductions ont principalement pour but de simplifier la lecture du livre ${ }^{14}$.

\section{II.4. Des différences marquées selon les pays: l'exemple franco-québécois}

Nous avons noté plus haut que les usages changeaient selon les pays. Ce qui semble inimaginable dans les langues européennes peut se produire dans d'autres langues, ainsi la traduction des noms de groupes de rock en hébreu: les Rolling Stones y sont les Ha'avanim Hamitagalgelot et les Beatles les Hah'ipushiot alors que personne en France n'oserait parler sérieusement des Pierres qui roulent ou des Scarabées. Les différences existent au sein même de l'Europe puisque les langues latines traduisent plus souvent les noms des figures historiques étrangères que les langues anglo-saxonnes (Newmark 1988: 215).

L'exemple de la Tchécoslovaquie communiste montre que les choix de traduction ont pu être politiques: on traduisait alors les noms des entreprises russes mais pas celles des pays capitalistes, ainsi que la RDA, qui devenait la NDR (Nemecká Demokratická Republika) alors que les USA demeuraient les USA (Elman 1986: 28).

Ces décisions politiques et/ou culturelles peuvent ainsi avoir plus de poids que le fait de parler une même langue comme le montrent, au sein de la francophonie, les approches diverses qui existent entre le Québec et la France.

En effet, pour des questions juridiques, liées par exemple aux articles 51 et $63^{15}$ de la Charte de la langue française, plusieurs types de noms propres doivent être traduits au Québec alors qu'il n'est pas nécessaire de le faire en France. 
Ces articles de loi obligent notamment certaines entreprises ${ }^{16}$ à traduire leur nom alors qu'aucune disposition juridique n'est prévue à cet effet en France. Ainsi, la chaîne américaine KFC (Kentucky Fried Chicken) a traduit son nom en PFK (Poulet Frit Kentucky) uniquement pour le Canada. D'autres restaurants proposent des versions bilingues telles que Mister Noodles / Monsieur Nouilles et Just Noodles / Juste Nouilles ${ }^{17}$. Les marques dont le nom est composé de noms communs anglais peuvent également présenter une traduction française (la coloration pour hommes Just for Men devient Just for Men / Pour hommes seulement).

Il n'y a finalement que peu de noms de marques traduits en France mais, comme nous le verrons ensuite avec les titres de films, les nouvelles versions ne sont pas obligatoirement identiques à celles du Québec. Ainsi, Mr. Clean est, depuis 1966, Mr. Propre en France et M. Net au Canada. On y voit également le goût français pour l'«anglais de synthèse» dans la traduction de Diet Coke (Coke Diète au Québec) en Coca-Cola Light. Nous n'avons en fin de compte découvert qu'une seule marque dont le nom ait été changé en France mais pas au Québec: Pier 1 Imports qui devient Pier Imports en France. Le nom commun pier ( «quai d'escale»), qui ne fait certainement pas partie de l'anglais de base des Français, semble être considéré dans cette nouvelle version comme un nom propre (nous assistons peut-être à une analogie avec le prénom Pierre?).

La modification en France en janvier 2002 du nom du service de courrier électronique de Yahoo ${ }^{18}$, Yahoo! Courrier en Yahoo! Mail ne serait pas possible au Québec pour les raisons juridiques précédemment citées, Yahoo! Courriel gardera certainement ce nom tant que les lois ne seront pas abrogées.

Les Français sont généralement surpris par les titres différents de plusieurs films américains ou anglais au Québec. Notre corpus est composé de films récents dont le titre original débute par les lettres $\mathrm{M}, \mathrm{N}$ et $\mathrm{O}$ du site $<$ http://www.films.qc.ca/films. $\mathrm{html}>$ en avril 2002. On peut voir un titre québécois calqué sur l'original (Monster's Ball devient Le bal du monstre au Québec et À l'ombre de la haine en France), parfois l'inverse (Mickey Blue Eyes traduit par Mickey les Yeux Bleus en France et Mickey Belle Gueule au Québec), mais le plus souvent on assiste à des multiples dénominations (Out of Sight et The Out-of-Towners deviennent respectivement Hors d'atteinte et Escapade à New York en France et Loin des regards et Les banlieusards arrivent en ville au Québec).

On assiste depuis une quinzaine d'années à une recrudescence de titres non traduits en France, un phénomène inexistant au Québec. Ainsi, Mad City, The Majestic, The Man Who Cried, Mansfield Park, Max Keeble's Big Move, Men in Black, Mimic, Mission to Mars, Mystery Men, Nurse Betty, Ocean's Eleven, On the Line, The One et The Other Sister ${ }^{19}$ ont gardé leurs titres originaux lors de leur sortie dans les salles françaises.

Les distributeurs français présentent un autre type de titre impensable au Québec: le titre modifié et «réanglicisé». Ainsi, dans notre corpus Not Another Teen Movie (Pas encore un film d'ados! au Canada) devient Sex Academy en France et Never Been Kissed (Un Baiser, enfin!) est transformé en Collège attitude, un titre qui reprend la syntaxe anglaise. On voit d'autres exemples hors de notre échantillon comme Split Second (1991), transformé en Killer Instinct. La même année avait vu le succès de Basic Instinct, et les distributeurs français ont probablement tenté d'établir une analogie entre ces deux films ${ }^{20}$. Le premier exemple que nous ayons découvert est le film de 
David Lynch, Eraser Head, qui s'est appelé Labyrinth Man en France à la fin des années 1970 avant de retrouver, avec le succès rencontré par le cinéaste, son titre original21 $\mathrm{Au}$ vu de la multiplication de ce type de titres, on imagine que donner des titres en anglais est un avantage pour les carrières commerciales des films, mais il est visiblement préférable que l'anglais proposé soit relativement simple, comme le montrent ces quelques exemples:

\begin{tabular}{|l|l|l|l|}
\hline Titre original & Titre français & Titre original & Titre français \\
\hline Children of the Corn & Horror Kid (1983) & Blue in the Face & Brooklyn Boogie (1995) \\
\hline Nightbreed & Cabal (1989) & Warriors of Virtue & Magic Warriors (1997) \\
\hline The Wizard & Vidéo Kid (1989) & Wild Things & Sexcrimes (1997) \\
\hline Relentless & Psychokiller (1989) & $\begin{array}{l}\text { The Myth of } \\
\text { Fingerprints }\end{array}$ & Back Home (1997) \\
\hline Company Business & Patriots (1990) & Hideous Kinky & Marrakech Express (1997) \\
\hline Body of Evidence & Body (1992) & I Went Down & Irish Crime (1997) \\
\hline Encino Man & California Man (1992) & East is East & Fish \& Chips (2000) \\
\hline Cool Runnings & Rasta Rockett (1993) & & \\
\hline
\end{tabular}

Cette volonté de présenter un titre dans un anglais relativement simple se retrouve dans des créations bilingues comme Killer, journal d'un assassin (1996) ou Docteurs in love (1982) (originellement Killer, a Journal of Murder et Young Doctors in Love). C. Truchot considère que «les distributeurs supposent une compétence en anglais de la part des consommateurs, mais pas de celle des anglophones. Ils préfèrent leur fabriquer un anglais de synthèse en prenant leurs sources dans la culture internationale médiatisée» (1990: 186). Excepté les longs-métrages anglophones, il n’y a, en France, que les films asiatiques qui héritent de titres anglais ${ }^{22}$ (par exemple City on Fire [1987], The Blade [1995] ou Violent Cop [1989]), les films européens seront soit traduits en français soit conservés dans leur langue d'origine comme Aprile (1997) ou Sogni d'oro (1981) de Moretti ${ }^{23}$.

Ce phénomène s'étend à la littérature. Le nombre de livres avec des titres anglais a considérablement augmenté ces dernières années en France (par exemple Football Factory de John King et L.A. Confidential de James Ellroy), même s'il s'agit généralement d'un anglais élémentaire ${ }^{24}$. Ainsi, Speed Queen de Stewart O'Nan n'a pas été modifié contrairement à ses romans plus récents The Names of the Dead ou A World Away (devenus Le nom des morts et Un monde ailleurs). De même, parmi les œuvres de Hanif Kureishi, seul Black Album n'a pas été adapté pour la France. Il est évidemment difficile de faire une comparaison avec le Québec en raison du peu de livres en anglais qui sont traduits par des maisons d'édition québécoises.

La traduction est également une pratique culturelle et, en tant que telle, il est logique que les choix soient divergents entre la France et le Québec. Sur le plan littéraire, B. Folkart (1986: 247) propose ainsi de naturaliser le toponyme danois Virum (que H. Martinet [1982] a traduit par les bien-pensants danois) en utilisant le XVI $I^{e}$ pour le public français et Outremont ou Westmount pour le public québécois.

Enfin, la proximité géographique avec les États-Unis a probablement des conséquences sur le traitement des noms propres. Les noms des équipes sportives américaines (Chicago Bulls par exemple) ne sont pas modifiés en France alors qu'ils sont adaptés, sans doute en raison de leur caractère familier, au Québec (les Bulls de 
Chicago). De même, les adaptations phonologiques ne sont pas identiques: les prononciations de Boston, Los Angeles ou Wisconsin sont plus francisées outre-Atlantique qu'en France - où l'on s'en tient à l'«anglais de synthèse» -, au contraire d'autres noms (Buffalo, Moncton) que les Québécois prononcent à l'américaine.

\section{TYPES DE NOMS}

Malgré l'importance du contexte historique et social, le rôle du type de nom est loin d'être négligeable. Ainsi, le cas des titres d'œuvres est particulier: en France, on a le plus souvent traduit les titres de livres, de films ou de symphonies mais jamais ceux de disques de variété, de rock et de jazz.

C'est particulièrement dans les projets de traduction automatique que la distinction entre les différents types de noms propres est cruciale. Il est nécessaire d'avoir un étiquetage plus fin que le simple $N P$ puisque les pratiques sont différentes selon le type de nom propre. Ainsi, comme le montre J. Podeur (1999: 73), le lycée Henri-IV se traduit en italien par liceo Henri-IV alors que la phrase acquis sous Henri $I V$ verra la modification du prénom: acquisito sotto Enrico IV.

Puisque cette partie se situe sous l'angle de jalons pour la traduction automatique, nous laisserons de côté le cas particulier de la traduction des ouvres artistiques, où tous les types de noms propres peuvent être modifiés (pour prendre un exemple célèbre, les noms de personnages comme Vendredi/Friday dans Robinson Crusoë) ou changés par des noms qui ne peuvent être considérés comme des équivalents que dans ce contexte précis (le Chemin des Dames cité dans la chanson $\mathrm{Zu}$ Potsdam unter den Eichen de Brecht et Weill devient Verdun dans sa traduction anglaise par John Willett). La nature ontologique des porteurs peut même être prise en compte, ainsi dans la version brésilienne des œuvres complètes de Balzac, les noms des personnes réelles ont été conservés, mais ceux des personnages (telle Eugênia Grandet) ont été portugalisés (Maillot 1979: 212).

Anthroponymes: Le fait que les noms de personnes ne se traduisent pas semble incontestable. Pourtant, il suffit de regarder un prénom courant comme Jean pour voir que les prénoms ont longtemps été adaptés ${ }^{25}$. Du prénom hébreu original, il existe pratiquement autant de déclinaisons qu'il y a de langues européennes (Giovanni, John, Ivan, Juan, Ion, Ian, Joan, Johannes, etc.). Ces changements ont été rendus problématiques par le passage dans d'autres langues. Ainsi, les missionnaires anglophones ont rencontré diverses versions de Bartholomew en swahili: Baritholomi, Bartolomayo, Bartholomayo, Baritolomayo, Baritholomayo, Bartolomeo et Balutolomayo (Nida 1964 : 194).

Ces adaptations ne se pratiquent plus aujourd'hui, si ce n'est dans des cas très particuliers. Les noms de rois ne sont plus adaptés (Juan Carlos en Espagne et non Jean-Charles); par contre, ceux des papes continuent de l'être (Jean-Paul II / Giovanni Paolo II / Jana Pawła II).

Un autre cas marginal concerne les noms des immigrants. Certains ont changé de nom, d'autres les ont adaptés aux réalités phonologiques de leur pays d'accueil ${ }^{26}$ (élision d'une partie du nom, etc.). Au Japon, on conseille aux personnes prenant la nationalité japonaise d'adopter un nom typiquement japonais. Peu d'Occidentaux deviennent japonais, mais Horiya (1999: 24) a recensé un Français nommé Claude Thierry qui a été naturalisé en 1977 sous le nom de Tiari Kukôdo. 
Il existe toutefois un type d'anthroponymes qui persiste à être traduit: le surnom. Si Sitting Bull est rarement modifié en France (alors que c'est une traduction en anglais de son nom) car il s'agit de son nom officiel, the Iron Lady a été traduit par la Dame de fer, the Great Helmsman par le Grand Timonier et plus récemment, Chemical Ali par Ali le Chimique. Nous n'avons rencontré qu'une exception célèbre: le surnom de Nicolae Ceausescu, le Conducator, était, il est vrai, plus transparent pour un francophone que ne l'est la version coréenne l'Éternel président, celui de Kim Il Sung. La preuve en est que d'autres surnoms du dirigeant roumain, tels le Danube de la pensée ou l'Étoile des Carpates, ont été traduits.

Toponymes: En ce qui concerne les toponymes, le facteur historique est primordial: les noms de pays vieux de plusieurs siècles sont modifiés en français (Espagne, Grande-Bretagne) alors que des noms plus récents (Vanuatu, Myanmar, Republika Srpska) ne le sont pas. Parmi les anciens, on peut dissocier ceux qui sont adaptés (France / Francia) et ceux dont une ou plusieurs parties sont tirées d'un nom commun qui se traduisent: Royaume-Uni / United Kingdom; New Zealand / Nouvelle-Zélande; G.D.R. est devenu R.F.A. en français / F.R.G. en anglais / N.D.K. en hongrois (Kálmán 1978: 102), la Côte d'Ivoire se dit Elfenbeinskysten en norvégien, Ivory Coast en anglais, H'of Hashenhav en hébreu, Costa do Marfim en portugais, etc.

Nous avons vu précédemment la différence de traitement entre les villes américaines et celles dont leur nom est tiré. Les noms des grandes villes sont en règle générale adaptés (München devient Munich en français, Monachium en polonais ou Monaco en italien), sauf dans quelques cas où la partie tirée d'un nom commun est traduite (New Orleans / Nouvelle-Orléans; Dar el-Béida / Casablanca; Le Cap / Kaapstad / Cape Town). Pour que le nom d'une plus petite ville soit modifié, il faut soit une raison historique (Bicocca / La Bicoque) ou géographique (pays bilingues ou régions frontalières). Ainsi, les villes alsaciennes ont des versions allemande et française (Saverne / Zabern; Mulhouse / Mülhausen, etc.) mais pas espagnole ou italienne car elles n'ont pas une grande importance pour les citoyens espagnols ou italiens. Certaines villes voient leur nom traduit officiellement (Mons / Bergen en Belgique) ou officieusement (Neufchâtel / Neuenburg en Suisse). On citera également Oxford ${ }^{27}$ qui se traduit en gallois par Rhydychen («ford»+ «oxen») (Evans et Wimmer 1990: 267).

Puisque la notoriété d'un toponyme est capitale pour qu'il soit modifié dans la langue cible, il en découle que tous les toponymes célèbres ou étant communs à plusieurs pays, c'est-à-dire les noms de continents, d'océans, de mers, de fleuves et de rivières importantes, etc., peuvent subir des adaptations (Antarctique / Antarctica) et des traductions. Ainsi, sous cet angle de la traduction, un francophone visitera en Italie la place Saint-Pierre (Piazza San Pietro) de Rome, la place Saint-Marc (Piazza San Marco) et le pont des Soupirs (Ponte dei Sospiri) à Venise; en Allemagne, la Forêt Noire (Schwarzwald), le Jardin anglais (Englischer Garten) de Munich, le Vieux Musée (Altes Museum) de Berlin; dans d'autres pays le Parlement (Országház) de Budapest, le pont Charles (Karlìv most) de Prague, la place Rouge (Krasnaja Ploščad') de Moscou et à Istanbul le Grand Bazar (Kapalı Çarşı) et le Bazar égyptien (Mısır Çarşı).

Les mers Blanche, Noire et Rouge gardent a priori les mêmes couleurs dans toutes les langues, le Great Salt Lake devient le Grand Lac Salé, la Tierra del Fuego se transforme en Terre de Feu en français et Feuerland en allemand, elle se traduit également en roumain mais non en anglais où l'on garde la version originale (Bantaş et Manea 1990: 186). Il existe bien d'autres types de toponymes pouvant être traduits (par 
exemple le Cap de Bonne-Espérance / Kap der Guten Hoffnung / Cape of Good Hope ou le Tropique du Capricorne / Wendekreis des Steinbocks / Tropic of Capricorn). Enfin, comme pour les anthroponymes, les surnoms sont généralement traduits (Big Apple / la Grosse Pomme).

M. Fourment-Berni Canani (1994: 556) estime qu'avec les couples software / logiciel et computer / ordinateur, il n'y a pas traduction mais nomination. Le cas des titres d'œuvres montre d'autre part qu'il est parfois difficile de parler de «traduction des noms propres». En fait, plus que d'une traduction, c'est à une appropriation culturelle que l'on assiste.

Il suffit de comparer deux cartes de pays différents pour s'apercevoir que de nombreux toponymes ont des noms distincts d'une langue à une autre ${ }^{28}$. Les sociolinguistes (en particulier Akin, 1999) pourraient nous dire que chaque groupe ethnique ou politique cherche à s'imposer par les noms. Dans les années 1980, les journaux argentins défendaient leurs Malvinas pendant que leurs homologues britanniques hurlaient contre l'invasion de leurs Falklands. De nombreuses îles ont ainsi deux noms totalement différents: les îles Tokdo (nom coréen) et Sakhaline (nom russe) sont respectivement appelées Takeshima et Karafuto par les Japonais. Au large de Bodrum, les Turcs ont sur leurs cartes l'île de Kardak alors que les Grecs y voient celle de Imia. Comme le note Maillot (1968b: 86), il est difficile de savoir que l'île croate de Krk prend le nom de Veglia en italien.

Diverses traductions officielles de toponymes sont donc loin d'être des calques. Les noms allemands du lac de Constance et de la Nouvelle-Bretagne (en PapouasieNouvelle-Guinée) sont ainsi Bodensee et Neupommern, et les îles des Princes au large d'Istanbul sont les «îles rouges» (Kızıl Adalar) en turc.

Selon Newmark (1981: 73), les toponymes que l'on retrouve à l'intérieur des villes (places, rues, etc.) ne se traduisent généralement pas. Nous en avons pourtant donné plusieurs spécimens plus haut et nous pourrions en donner d'autres, les noms de rues de New York sont par exemple parfois traduites comme dans cet extrait d'un article du magazine Octopus ( $\mathrm{n}^{\circ} 20$, janvier/février 2003): "C'était en 68, à l'époque où j'étais carilloneur à l'église Saint Thomas à New York, au coin de la $53^{\mathrm{e}}$ et de la $5^{\mathrm{e}}$ avenue.» La question du bilinguisme peut également se faire ressentir au niveau des odonymes, les panneaux de la commune de Hamsptead dans la banlieue de Montréal donnent ainsi les versions dans les deux langues: "Chemin Queen Mary Road», «Rue Fichley Road» et "Place Heath Place». La syntaxe des deux langues permet de faire cohabiter les deux versions sur un même panneau, mais cet exemple prouve aussi que la traduction automatique peut éprouver des difficultés face à des cas a priori simples puisque road se traduit une première fois par chemin et une autre par rue.

On peut toutefois penser que la traduction automatique des toponymes est sans doute l'une des moins problématiques, la liste des toponymes modifiables dans la langue cible est plus ou moins finie, même si quelques cas d'homonymies compliquent la tâche: la Red River qui se jette dans le Mississipi n'a pas de version française, au contraire de celle qui est tributaire du lac Winnipeg (rivière Rouge).

Noms de l'astronomie: Comme les toponymes connus depuis longtemps, les noms de planètes sont traduits, les autres, comme Milky Way / Voie lactée, Pole Star / Étoile polaire ou les noms de constellations (le Bélier / the Ram / der Widder), le sont généralement. 
Noms de monuments: On pourrait penser de manière prosaïque que, plus un monument est visité par des touristes étrangers, plus il a de chances de voir son nom traduit (tour Eiffel / Eiffel Tower; Den Lille Havfrue / La Petite Sirène de Copenhague). Il ne s'agit pourtant pas d'une règle infaillible puisque Big Ben, le Golden Gate, le Taj Mahal ou encore le Manneken Pis ne sont pas modifiés en français, alors que leur nom est facilement traduisible (manneken signifie "petit bonhomme» en flamand, mahal «palais» en hindi, etc.).

Imitations de noms propres: Par «imitation de noms propres», nous pensons à tous ces noms tels John Doe ou Trifouilly-les-Oies qui servent à référer à un quidam ou à un lieu quelconque. Ces noms, qui ne sont pas interlinguaux doivent le plus souvent être traduits par leurs équivalents dans les langues-cibles ou par des périphrases.

Noms mythologiques: Ces noms sont généralement traduits ou adaptés comme les noms de la mythologie scandinave (Thor, Odin, etc.). Le christianisme a également eu besoin de traduire le nom de Dieu dans les pays où il cherchait à s'implanter, on voit ainsi dans les bibles d'Afrique sub-saharienne des Allah tout au long du texte (Loewen 1985: 203) ou encore Prabhu («seigneur») en hindi (Rai 1992: 445).

Noms de peuples: Puisqu'ils existent également sous la forme d'adjectifs par exemple, ils sont logiquement toujours traduits.

Noms d'animaux: Ces noms ne sont $a$ priori pas traduits, les exceptions remarquées relevant de la littérature enfantine (Milou devient Snowy dans la version anglaise des aventures de Tintin).

Noms d'artefacts: Que ce soit les noms d'avions (Spirit of St Louis) ou de navettes spatiales (Challenger), aucun ne semble être traduit en français. Parmi les divers objets ayant un nom propre, peu se traduisent: la Pierre de Rosette / Rosetta stone est une exception face à Little Boy (la bombe d'Hiroshima), Big Ben ou the Union Jack. En ce qui concerne les pierres précieuses, si le Koh-e-noor n'est pas modifié, ce n'est pas le cas de la Panthère rose / Pink Panther.

Noms de marques: La plupart des entreprises tentent aujourd'hui d'avoir des noms facilement prononçables dans toutes les langues, on assiste en particulier à une propagation du latin, du grec et de l'anglais (Raab Karcher Energieservice est devenu Viterra Energy Services [contraction de vita et terra], Vivendi, Vinci, Thales, Accenture). Les modifications sont donc très rares mais il en existe quelques-unes (La Vache qui rit / The Laughing Cow / Die lachende Kuh).

Noms d'enseignes: Ce type de noms n'est généralement pas traduit, mais il est possible qu'un nom commun faisant partie du nom (hôtel, restaurant, etc.) le soit.

Titres de journaux ou d'émissions: Tous les manuels de traduction diront qu'ils ne sont jamais traduits (The Guardian, die Welt, etc.), pourtant on peut voir sur le site d'information anglais Ananova que les noms des quotidiens chinois sont traduits en anglais (par exemple Daily Sunshine).

Titres d'œuvres: Darbelnet (1977: 14) avait remarqué qu'une pièce américaine pouvait avoir deux titres dans la même langue, l'un pour New York et l'autre pour Londres et, qu'à plus forte raison, il fallait accepter que ce titre soit différent dans une autre langue. Comme nous l'avons vu avec les titres de films et ceux de disques, les conventions ne sont toutefois pas les mêmes selon la nature de l'œuvre (ou le style musical): si les titres de tableaux (Les Ménines ou Les Fileuses de Vélasquez) sont généralement traduits et Das Lied von der Erde de Mahler devient Le Chant de la terre en français, les titres d'albums de rock ne seront pas modifiés ${ }^{29}$. 
Phénomènes climatiques: Les noms d'ouragans ou de tempêtes sont conservés dans leur forme originale, ce qui découle du fait qu'ils sont tirés de prénoms. Les autres types de noms tels Gulf Stream ou El Nino le sont également.

Périodes historiques: Ces noms sont généralement traduits, Gläser (1989: 75) cite The Franco-Prussian War / Der Deutsch-Französische Krieg et The Glorious Revolution / Die Glorreiche Revolution, on pourrait ajouter le Traité de Versailles / Versailles Treaty et Gulf War / Guerre du Golfe. Les noms comme l'Anchluss qui sont conservés sous leur forme originale sont très rares en français, mais plus courants en anglais (Kristallnacht).

Les événements historiques reçoivent également des appellations différentes selon les camps. Dans son article Sens et dénotation, Frege fait ainsi allusion à la bataille de la Belle Alliance, celle que l'on nomme Waterloo en France. Les connotations sont bien dissemblables puisqu'un Français perçoit difficilement la «beauté» de cette alliance.

Associations, partis, institutions, etc. : Delisle (1993: 415) estime que ces noms sont le plus souvent traduits lorsque les mots qui les composent sont des noms communs: National Film Board / Office national du film.

En fait, ces noms doivent être traduits dans les pays bilingues: Royal Canadian Mounted Police / Gendarmerie Royale du Canada (et non la police montée); SNCB (Société Nationale des Chemins de fer Belges) devient en flamand NMBS (Nationale Maatschappij der Belgische Spoorwegen). Les associations internationales tentent de posséder des versions adaptées à chaque langue (Médecins Sans Frontières / Doctors Without Borders, Croix Rouge / Red Cross). Pour les pays unilingues comme la France, le choix est plus libre. Si ces noms sont généralement traduits (la bande à Baader, la Bande des quatre, le Parti communiste bulgare, les Panthères noires, l'IRA-véritable, le Cercle de Vienne, l'Armée rouge, etc.), on note ces dernières années une montée des noms non modifiés (Tsahal, Batasuna, Al-Qaeda, Jamaa Islamya).

Enfin, certains noms peuvent être traduits dans des contextes particuliers, ce que fait Anne Reboul lors d'un colloque lorsqu'elle se présente comme faisant partie de l'«Institute for Cognitive Sciences, CNRS, France».

Divers: Les noms de politiques comme la NEP ou le New Deal ne sont pas modifiés en français, contrairement aux noms d'opérations militaires célèbres (plan Barbarossa). L'un des noms propres les plus récents, Web, a déjà trouvé un calque en français avec la Toile.

\section{CONCLUSION}

L'idée que les noms propres sont intraduisibles est largement partagée en linguistique, du moins chez ceux qui ne s'intéressent pas directement à la traduction. La vision étroite des noms propres qu'ont de nombreuses personnes en est une des raisons principales: les noms propres ne se limitent pas aux anthroponymes et aux toponymes, il est important de le répéter. Les noms de partis politiques, d'événements historiques et particulièrement tous les types de surnoms, sont le plus souvent traduits.

Devant la difficulté de trouver une description satisfaisante du nom propre, Mańczak (1968) a choisi comme critère définitoire l'intraduisibilité et ajoute même que sa définition est préférable aux autres parce qu'elle n'est pas logique mais linguistique. Sans parler du risque de considérer tout nom intraduisible comme un nom propre, il est évident à la lumière des exemples que nous venons de donner que le 
linguiste polonais fait fausse route. Les choix de modification ou de conservation des noms propres dans la langue cible peuvent être culturels (le nom de la Katholieke Universiteit Leuven n'est pas modifié en anglais ${ }^{30}$ alors qu'il l'est en français), commerciaux (la propension des entreprises à adopter ces dernières années des noms interlinguaux pour éviter toute traduction), stylistiques (rôle du genre textuel), politiques (comme l'interdiction de naturaliser le nom du Myanmar), en fonction du degré de familiarité avec la langue source (un nom propre anglais n'est pas traité de la même manière qu'un nom propre chinois ou russe), etc., mais très rarement linguistiques. La comparaison entre la France et le Québec montre également que l'intraduisibilité ne peut être une définition adéquate: les différences politiques, juridiques et culturelles entre les deux pays ont plus de poids dans la décision finale que la langue commune.

La traduction est une pratique culturelle et, puisque nous avons tendance à accorder plus de poids au nom propre qu'aux autres catégories de mots, sa traduction est devenue particulièrement complexe car il est nécessaire de jongler entre les divers facteurs que nous avons cités. La tendance actuelle en français est de suivre le modèle anglo-saxon qui consiste à conserver le maximum de formes originales. Il est possible de s'en réjouir ou de le regretter à l'instar de Pichon (1937) et Schafer ${ }^{31}$ (1954), mais l'utilité d'établir un cadre plus strict pour éviter les trop fréquentes erreurs de traduction est patente. Ces erreurs sont de natures diverses, quelques-unes dues aux différences de traitement chronologiques (le nom de la ville argentine de Cordoba modifié en Cordoue sur Educnet, un site du ministère de l'Éducation), d'autres au passage par une tierce langue (D. Olivier [1966:4] recense une traduction de la Bible faite sur la base de l'allemand et qui a négligé le fait que le Pentateuque s'appelle Moses en allemand: on lit donc Moïse 1, Moïse 2, etc. pour la Genèse, l'Exode, etc.) ou encore des erreurs par rapport au type de nom: malgré le fait que les noms de disques de rock ne se traduisent normalement pas, on entend dans la version française de High Fidelity (2001) un des protagonistes dire «cela va me permettre de vendre cinq exemplaires des trois singles du Beta Band », alors que le disque en question s'intitule The Three E.P.'s.

Ce dernier exemple prouve que tous les types de noms propres ne doivent pas être abordés de manière uniforme; cependant, l'établissement d'une ontologie regroupant les diverses sortes de noms propres ne résoudrait pas les difficultés liées aux différences de contextes. La question primordiale, avant de penser à traiter convenablement les noms propres par des machines, serait justement de savoir comment les programmes pourront prendre en compte le contexte, et la réponse à cette question reste des plus incertaines.

\section{NOTES}

1. Algeo fait également un rapprochement entre les noms propres et les mots d'emprunt comme "dharma» en anglais: "The subsequent use of all such loan words as equivalents for their etyma must be called translation, unless an arbitrary and a priori terminological distinction is to be made» (1973: 62).

2. Nous préférons pour plus de simplicité nous limiter à trois possibilités plutôt que d'adopter la typologie plus fine de Nida (1964) qu'a adoptée Grass (2002).

3. Le nom de cette école est Holy Cross. Plusieurs reportages télévisés ont également employé ce nom traduit en français.

4. On notera toutefois que El Greco est devenu Le Greco en français et non Le Grec. 
5. L'argument de la fréquence qu'invoque Ballard (2001:29), et qui expliquerait pourquoi London en Ontario n'est pas traduit, est faux. Ce nom est très souvent employé par les Canadiens francophones, peut-être plus chez certains que celui de la capitale anglaise.

6. Cependant, Athens est la forme anglicisée et non l'originale.

7. D'autres langues d'Europe de l'Est continuent d'adapter les noms des personnalités. Ainsi, d'après Beci (2001: 28), on voit en albanais les formes Shekspir, Sharl de Gol et Nju-Jork. De même, en serbocroate, on traduira «Nous avons vu Louis de Funès» par «Gledali smo Luj de Finesa (Osipov 2001: 251).

8. Dans la version italienne de Gone With the Wind (1937), Rossella O'Hara aime Ashley Wilkes mais se marie avec Carlo Hamilton, Franco Kennedy et Rhett Butler. On voit que les prénoms qui ont des équivalents italiens sont naturalisés, alors que les autres sont conservés.

9. Parallèlement à ces abandons des formes francisées, on voit disparaître les accents ajoutés en français (Breda ne s'écrit plus Bréda comme dans le tableau de Vélasquez La Reddition de Bréda) et, inversement, les signes diacritiques originaux tendent à être rendus en français (Málaga et non plus Malaga dans Le Petit Robert des noms propres).

10. Les germanophones ont ainsi maintenu le nom italien Nizza (ce qui est logique pour une ville longtemps italienne) au détriment de Nice.

11. Un phénomène identique est observable avec quelques noms communs. Ainsi, le mot hébreu shekel sera traduit par sicle dans un contexte biblique, mais ne sera pas modifié lorsqu'il s'agit de la monnaie israélienne actuelle. Les emprunts posent un problème proche: Gestalt sera traduit selon les contextes par forme ou par gestalt.

12. Il y a toutefois deux autres exceptions, le Musée de Navarre de Pampelune et le célèbre Musée $d u$ Prado de Madrid sont donnés en version française mais pas dans leur version originale.

13. On notera au passage que British devient ici anglais. Le guide de la Grande-Bretagne utilise une autre technique qui est, dans le cas du Museum of Science and Industry de Manchester, de donner la traduction en légende de la photographie mais pas dans le corps du texte.

14. Un autre exemple tiré de ce livre montre que les choix de traduction ou de non-traduction ne sont pas systématiques: «On lit plein de magazines, au poste. Le chef est abonné à Cinéfan, 16/9, et les Dessous d'Hollywood, Carl à Médiazone, et moi à Travelling, Film Finger et People» (Stephen Wright, États sauvages, p. 128).

On aperçoit un cas similaire dans un livre de Benjamin (Euvres I- Mythe et violence, Paris, Denoël, 1971, p. 105) où le titre de l'article est traduit mais pas le nom de la revue: «Dans son étude sur le Langage dans la structure de la civilisation totale, Leo Weisgerber - actuel éditeur de Wörter und Sachen - s'est intéressé de façon détaillée aux corrélations entre le langage et la civilisation matérielle.»

15. Article 51: «Toute inscription sur un produit, sur son contenant ou sur son emballage, sur un document ou objet accompagnant ce produit, $\mathrm{y}$ compris le mode d'emploi et les certificats de garantie, doit être rédigée en français.»

Article 63: «Le nom d'une entreprise doit être en langue française.»

16. Il est utile de prendre en compte le fait que les noms d'entreprises ont pour diverses raisons des types de construction différents. Pour reprendre un titre de film, on notera que Monsters, Inc. devient Monstres, inc. au Québec et Monstres \& cie en France. De même, la branche canadienne de General Motors se nomme General Motors du Canada Limitée et la française General Motors France. Les termes incorporée et limitée, empruntés à l'anglais américain, sont totalement absents de la juridiction française.

17. On peut toutefois se demander s'il s'agit réellement de français ou plutôt d'un «français de synthèse».

18. Ce choix semble lié à la décision de Yahoo d'uniformiser les noms de ses services de courrier. Si l'on excepte l'Argentine, le Mexique et l'Espagne qui partagent Yahoo! Correo et la Corée qui propose un service dont le nom est écrit en caractères coréens, tous les autres pays (Allemagne, Italie, Grèce, Brésil, Suède, Norvège, Danemark et même la Chine et le Japon,) ont uniquement le service Yahoo! Mail en caractères latins.

19. Respectivement Reportage en direct, Le Majestic, L'homme qui pleurait, Lettres de Mansfield Park, Le Grand Coup de Max Keeble, Hommes en noir, Métamorphose, Mission sur Mars, Les Supposés Héros, Garde Betty, L'inconnu de Las Vegas, En bout de ligne, Le Seul et L'Autre sæur au Québec.

20. De même, Strictly Ballroom (1991) devient Ballroom Dancing en référence à Dirty Dancing. Le procédé est utilisé pour des films à petit budget. Ainsi, un téléfilm australien sur un boxeur aborigène qui s'intitule Rose against the Odds (1992) est devenu Crocodile Champion, en référence à Crocodile Dundee, le long-métrage australien ayant connu le plus gros succès en France. 
21. Freaks (1932) a eu pour titre français La Monstrueuse Parade avant de retrouver son titre anglais il y a quelques années.

22. Il semblerait que les producteurs asiatiques distribuent internationalement leurs films avec des titres anglais.

23. Les films slaves, scandinaves, germanophones, iraniens, indiens, etc. sont dans une écrasante majorité traduits en français et nous n'avons même jamais vu dans les répertoires des titres tirés du chinois ou d'une langue africaine. Nous contrebalancerons tout de même ces propos en notant l'apparition récente de quelques titres en japonais, par exemple Hana-Bi (1997) ou Kaïro (2001).

24. Un des principaux contre-exemples vient du roman Queer de Burroughs, car ce mot ne fait pas partie de la connaissance de base des Français. Il faut peut-être en chercher la raison dans le fait que ce livre était la suite de Junky, dont le titre original avait également été conservé.

25. Kalverkämper (1978: 88) écrit qu’on ne traduit pas Johann Sebastian Bach en Jean Sébastien Ruisseau. Ce n'est pourtant que partiellement vrai car son prénom est généralement francisé, et la prononciation de son nom est souvent très éloignée de l'originale.

26. Si l'adaptation est une pratique bien plus courante que la traduction chez les immigrés américains, Adamic (1942: 47) a noté quelques exceptions au début du xx siècle: le Grec Marangopoulos a traduit littéralement son nom en Carpenter, le Français Bienvenue en Welcome, le Slovène Zelenjak et le Lituanien Zalionis en Green, le Polonais Piekarz en Baker. Betten et Du-nour (1998: 271) citent également le cas d'un immigré qui, dans le cadre de la campagne d'hébraïsation des noms en Israël, a traduit son patronyme Levisohn en Bar-Levi.

27. Oxford est traduit d'une manière identique en chinois: Niujin signifie «le gué aux bœufs» (Alleton 1993: 227).

28. Les langues peuvent aussi offrir des distinctions qui n'existent pas ailleurs. Ainsi, le néerlandais découpe le Rhin en plusieurs segments: Kromme Rijn («Rhin tordu»), Oude Rijn («vieux Rhin»), Nieuwe Rijn («nouveau Rhin») selon la partie de son cours.

29. À l'instar des autres types de noms propres, ils pourront être modifiés dans certains contextes particuliers. Ainsi, lors d'une interview (The Wire, $n^{\circ} 232$, juin 2003), David Sylvian traduit le titre de la chanson Der Osten ist Rot de Holger Czukay en The East is Red.

30. Un appel à communication en anglais pour une conférence annonce toutefois que l'événement sera organisé par le «Centre for Computational Linguistics of the Katholieke Universiteit Leuven». Au contraire du second qui n'est pas modifié, le premier nom est la traduction de Centrum voor Computerlinguïstiek.

31. Cet auteur est sinologue et déplore la traduction en anglais d'une porte par Ying t'ien Gate. Pour lui, le nom devrait être traduit par Gate of Responsiveness to Heaven, le lecteur non sinophone comprendrait ainsi la même chose qu'un Chinois. Le contenu de nombreux noms chinois est clair, mais la traduction impose une opacité inutile.

\section{RÉFÉRENCES}

Adamic, L. (1942): What's your Name?, New York, Harper and Brothers.

AкIN, S. (1999) : «Pour une typologie des processus redénominatifs», in S. AKIN (dir.): Noms et re-noms: la dénomination des personnes, des populations, des langues et des territoires, Rouen, Publications de l'Université de Rouen, p. 33-60.

Algeo, J. (1973): On Defining the Proper Name, Gainesville, University of Florida Press.

Alleton, V. (1993): Les Chinois et la passion des noms, Paris, Aubier.

Ballard, M. (2001): Le nom propre en traduction, Gap, Ophrys.

Bantaş, A. and C. Manea (1990): «Proper Names and Nicknames: Challenges for Translators and Lexicographers", Revue roumaine de linguistique 35-3, p. 183-196.

BECI, B. (2001) : «Des caractéristiques grammaticales du nom propre en albanais», Cahiers balkaniques 32, p. 17-29.

Betten, A. und M. Du-Nour (1998): Wir sind die Letzten. Fragt uns aus, Gerlingen, Bleicher.

CArton, F. (1968): «De la prononciation des noms propres», La Linguistique 4-2, p. 135-141.

Darbelnet, J. (1977): «Niveaux de la traduction», Babel 23-1, p. 6-17.

DAUZAT, A. (1928): Les noms de personnes - origine et évolution, Paris, Delagrave.

Dauzat, A. (1977): Les noms de famille de France, Paris, Guénégaud.

Delisle, J. (1993): La traduction raisonnée - Manuel d'initiation à la traduction professionnelle anglais $\rightarrow$ français, Ottawa, Presses de l'Université d'Ottawa. 
Demonet, M.-L. (1992): Les Voix du signe - Nature et origine du langage à la Renaissance (14801580), Paris-Genève, Champion-Slatkine.

Elman, J. (1993): «Le problème de la traduction des noms propres», Babel 32-1, p. 26-30.

Evans, M. J. and R. Wimmer (1990): «Searle's Theory of Proper Names, From a Linguistic Point of View», in A. Burkhardt (éd.): Speech Acts, Meaning and Intentions - Critical Approaches to the Philosophy of John R. Searle, Berlin, Walter de Gruyter, p. 259-278.

Folkart, B. (1986) : «Traduction et remotivation onomastique», Meta 31-3, p. 231-252.

Fourment-Berni Canani, M. (1994): «Le statut des noms propres dans la traduction», Studi italiani di linguistica teorica e applicata 23-3, p. 553-571.

Gläser, R. (1989) : «Zur Übersetzbarkeit von Eigennamen», in F. Debus \& W. Seibicke (éd.): Reader zur Namenkunde - I. Namentheorie, Hildesheim, Georg Olms, p. 67-78.

Grass, T. (2002): Quoi! Vous voulez traduire "Goethe»? - Essai sur la traduction des noms propres allemand-français, Berne, Peter Lang.

Hermans, T. (1988): "On Translating Proper Names, with reference to De Witte and Max Havelaar", in M.J. Wintle (éd.): Modern Dutch Studies - Essays in Honour of Peter King, Professor of Modern Dutch Studies at the University of Hull on the occasion of his retirement, Londres, The Athlone Press, p. 11-24.

Horiya, H. (1999): Nomination des personnes en japonais, Mémoire de DEA, Montpellier, Université Montpellier III.

Humbley, J. (2000) : «Le titre, catégorie de nom propre?», BULAG, $\mathrm{n}^{\circ}$ hors-série: Lexique, syntaxe et sémantique - Mélanges offerts à Gaston Gross à l'occasion de son $60^{\circ}$ anniversaire, p. 309320.

KÁlmán, B. (1978): The World of Names - A Study in Hungarian Onomatology, Budapest, Akadémiai Kiadó.

KALVerkäMPER, H. (1995) : «Namen im Sprachaustausch: Namenübersetzung», in E. Eichler et al. (éd.): Namenforschung / Name Studies / Les Noms propres, Tome 2, Berlin-New York, Walter de Gruyter, p. 1018-1025.

Loewen, J. A. (1985): «Translating the Names of God: How to Choose the Right Names in the Target Language», The Bible Translator 36-2, p. 201-207.

Maillot, J. (1968): «Toponymie et traduction (suite)», Babel 14-2, p. 86-91.

Maillot, J. (1979): «Anthroponymie et traduction», Babel 25-4, p. 210-213.

Manini, L. (1996): «Meaningful Literary Names - Their Forms and Functions and their Translation", The Translator 2-2, p. 161-178.

MaŃCZAK, W. (1968) : «Le nom propre et le nom commun», Revue Internationale d'Onomastique 20-3, p. 205-218

Martinet, A. (1982): «La classe des noms propres en français et ailleurs», Glossologia 1, p. 716.

Newmark, P. (1981): Approaches to Translation, Oxford, Pergamon Press.

Newmark, P. (1988): A Textbook of Translation, New York, Prentice Hall.

NidA, E. A. (1964): Toward a Science of translating - With Special Reference to Principles and Procedures Involved in Bible Translating, Leiden, E.J. Brill.

Olivier, D. (1966): «Pièges des traductions bibliques», Traduire 45, pp.3-6.OSIPOV Vladimir (2001): "Notes onomastiques du domaine "serbo-croate" ", Cahiers balkaniques 32, p. 229253.

Pichon, E. (1937): «L'appellation française des lieux étrangers», Le français moderne 5-2, p. 113120.

Podeur, J. (1999) : Nomination in azione - il nome proprio nelle traduzioni dall'italiano al francese e dal francese all'italiano, Naples, Liguori.

RAI, B. (1992): «What is His Name? - Translation of Divine Names in Some Major North Indian Languages", The Bible Translator 43-4, p. 443-446.

Rey, A. (1979): La terminologie: noms et notions, Paris, PUF.

Rey-Debove, J. (1969): «Le signe et la chose dans le discours métalinguistique», Travaux de linguistique et de littérature 7-2, p. 113-129. 
738 Meta, LI, 4, 2006

Salmon, N. (1989): «How to Become a Millian Heir», Noûs 23-2, p. 211-220.

Schafer, E. H. (1954) : «Non-Translation and Functional Translation-Two Sinological Maladies», The Far Eastern Quarterly 13-3, p. 251-260.

Schultze, B. (1991): «Problems of Cultural Transfer and Cultural Identity: Personal Names and

Titles in Drama Translation", in H. Kittel \& A.P. Frank (éd.): Interculturality and the Historical Study of Literary Translations, Berlin, Eric Schmidt, p. 91-110.

TRUснот, C. (1990): L'anglais dans le monde contemporain, Paris, Le Robert.

Vaxelaire, J. L. (2001): Pour une lexicologie du nom propre, Thèse de doctorat, Paris, Université Paris VII. 